\title{
Disconnection from others in autism is more than just a feeling: whole-brain neural synchrony in adults during implicit processing of emotional faces
}

\author{
Rocco Mennella $a^{1,2^{*}}$ (D) Rachel C. Leung ${ }^{1,5}$, Margot J. Taylor ${ }^{1,3,4,5}$ and Benjamin T. Dunkley ${ }^{1,3,4}$
}

\begin{abstract}
Background: Socio-emotional difficulties in autism spectrum disorder (ASD) are thought to reflect impaired functional connectivity within the "social brain". Nonetheless, a whole-brain characterization of the fast responses in functional connectivity during implicit processing of emotional faces in adults with ASD is lacking.

Methods: The present study used magnetoencephalography to investigate early responses in functional connectivity, as measured by interregional phase synchronization, during implicit processing of angry, neutral and happy faces. The sample $(n=44)$ consisted of 22 young adults with ASD and 22 age- and sex-matched typically developed (TD) controls.

Results: Reduced phase-synchrony in the beta band around 300 ms emerged during processing of angry faces in the ASD compared to TD group, involving key areas of the social brain. In the same time window, de-synchronization in the beta band in the amygdala was reduced in the ASD group across conditions.

Conclusions: This is the first demonstration of atypical global and local synchrony patterns in the social brain in adults with ASD during implicit processing of emotional faces. The present results replicate and substantially extend previous findings on adolescents, highlighting that atypical brain synchrony during processing of socio-emotional stimuli is a hallmark of clinical sequelae in autism.
\end{abstract}

Keywords: Autism, Functional connectivity, Magnetoencephalography, Social brain, Emotional faces, Young adults

\section{Background}

Socio-emotional deficits represent a core symptom in autism spectrum disorder (ASD) and are thought to have modulatory effects over other clinical manifestations [1]. It has been proposed that those with ASD show a lack of motivation toward social stimuli, such as emotional faces, which are considered to be naturally rewarding in typically developed (TD) individuals [1-3]. Other authors related socio-emotional difficulties in ASD to a deficit in theory of mind [4], or to a pervasive difficulty in learning stimuli's emotional salience [5]. In the past years, different

\footnotetext{
*Correspondence: rocco.mennella@gmail.com

'Department of Diagnostic Imaging, Hospital for Sick Children, 555 University Avenue, Toronto, Ontario M5G 1X8, Canada

${ }^{2}$ Department of General Psychology, University of Padova, Via Venezia 8, 35131 Padova, Italy

Full list of author information is available at the end of the article
}

theoretical approaches have highlighted atypical activation of specific brain regions in individuals with ASD, such as the amygdalae $[6,7]$, insulae $[8,9]$, fusiform gyri [1] or superior temporal sulci [10]. Nonetheless, recently it has been suggested that ASD can be described as a neural systems disorder [11], characterized by widespread abnormalities throughout the brain [12].

Specifically, dysfunctional emotional processing and an impaired ability to recognize others' emotions correctly have been related to disturbances in functional connectivity among brain areas important to assessing stimuli's salience [13, 14]. In autism, this is thought to reflect atypical maturation of the "social brain", a network of brain areas which shows co-activation across social tasks $[15,16]$. The social brain includes limbic and paralimbic regions which code for different aspects of emotional 
significance of social stimuli, such as the amygdalae [17] and anterior hippocampi [18], the anterior insulae [19], the medial and ventromedial prefrontal cortices [16], and the anterior temporal lobes [20]. Other areas in the social brain are involved in representing shape, movement and conceptual information about animate entities (i.e. the fusiform gyri, the posterior cingulate/precuneus and regions within and near the posterior superior temporal sulci) $[1,21]$, in action understanding (i.e. somatosensory and anterior intraparietal cortices) [22] and social communication (i.e. the left inferior frontal gyrus) [23] (for a review see [24]). Studies using functional magnetic resonance imaging (fMRI) have reported impaired (usually decreased) connectivity in the social brain network in individuals with ASD at rest $[24,25]$, during processing of faces [26] and emotional faces [27, 28]. Recent evidence suggested that hypo-connectivity in autism is more evident during implicit compared to explicit emotional processing, and explicit emotional processing may be relatively preserved in high-functioning individuals with ASD due to learning and experience [28]. Since it is well known that implicit processing of emotional stimuli, such as emotional faces, relies on quick and automatized processes in the brain [29-31], a precise characterization of the time course of early brain activations in ASD is very relevant to understanding socio-emotional processing in ASD.

Compared to fMRI, magnetoencephalography (MEG) provides complementary information about the timing of the neural response, while still maintaining a good spatial resolution [32, 33]. Moreover, MEG allows the investigation of connectivity patterns at different frequency scales, which are differently modulated by emotional dimensions. In particular, emotional valence (pleasant/ unpleasant) is usually represented by stimulus-related changes in higher frequencies (beta/gamma) in early time windows, whereas arousal is reflected by lower frequencies [34-42]. MEG and EEG studies consistently report beta band modulation during both explicit and implicit emotion recognition and understanding of social-emotional stimuli $[43,44]$. During implicit emotional processing of dynamic facial expressions, synchronization in the beta band between frontolimbic (i.e. pulvinar, caudate, cingulate, and amygdala) and posterior regions of the social brain (i.e. superior temporal sulcus) was observed when the facial expressions become emotionally salient [44]. This finding, along with the observed spatially concomitant fMRI BOLD response, led to the interpretation that the beta rhythm subserves feed-forward/feed-back mechanisms between fronto-limbic and posterior areas during emotional processing of socially relevant stimuli. Moreover, several studies of implicit emotional processing of faces using both intracranial recordings [45] and MEG
$[46,47]$ suggest a role for fast gamma synchronization in subcortical limbic regions (e.g. thalamus and amygdala) in response to negatively valenced faces, followed by synchronization in both frontal and posterior areas. Accordingly, gamma synchrony has been successfully employed in assessing thalamo-cortico-limbic circuitry during implicit emotional processing of facial expressions [48].

Thus, the study of oscillatory responses in the beta and gamma ranges reliably characterizes fast spatiotemporal coupling in the social brain in response to emotional stimuli. Importantly, consistent results also come from several studies investigating implicit emotional processing of facial expressions. This is particularly relevant for the study of autism, since, as mentioned above, fMRI studies have indicated that hypo-connectivity in autism is more evident during implicit compared to explicit emotional processing [28]. Two MEG studies have examined functional connectivity during emotional face processing in ASD, and both employed an implicit emotional paradigm. Khan et al. [49] investigated MEG functional connectivity involving the fusiform gyri, in adolescents and young adults with ASD. During processing of fearful and angry faces, reduced connectivity within the right fusiform gyrus and between the fusiform and the left precuneus, left inferior frontal gyrus and left anterior cingulate was reported in ASD, within 320 ms. In accordance with these results, decreased connectivity in beta band was also seen in adolescents with ASD during implicit processing of angry faces, in the first $400 \mathrm{~ms}$ after the stimuli [41]. In the latter study, reduced beta connectivity in ASD was anchored in connections between visual (e.g. the fusiform) and limbic (e.g. the insula) areas involved in face-processing, consistent with the role of beta band in coupling limbic and posterior visual activation [44].

\section{The present study}

Although there is support for early MEG underconnectivity in adolescents with ASD compared to TD, especially during processing of negatively valenced faces, a whole-brain characterization of MEG connectivity pattern in adults with ASD is lacking. Thus, in line with previous studies, we used an implicit emotional face paradigm [41] to investigate patterns of connectivity in young adults with ASD in response to angry, neutral and happy faces. Based on prior findings, we expected:

- A fast task-induced increase in connectivity in high-frequency bands (beta/gamma) in both groups [41, 42];

- Reduced connectivity within 400 ms in ASD vs. TD individuals between areas of the social brain [41, 49-51]. 


\section{Methods}

\section{Participants}

Forty-four young adults were included in the study, 22 with ASD (7 females, age $=26.4 \pm 4.1$ ) and 22 TD controls ( 8 females, age $=26.0 \pm 3.9$ ), matched for age and sex. All subjects had no history of neurological or neurodevelopmental disorder (other than autism), no standard contraindication for MEG and MRI, IQ $\geq 70$, language skills adequate for task comprehension and normal or corrected to normal visual acuity. As is typical in clinical samples, the majority of ASD participants were taking standard psychotropic medications at the time of the study (e.g. Ritalin, antidepressants and anxiolytics), while controls were free from medication. All participants read and signed an informed written consent, after receiving a complete description of the study. Testing and recordings were completed in the MEG Lab at the Hospital for Sick Children, and the Research Ethics Board of the hospital gave institutional approval for the study.

\section{Clinical evaluation}

The diagnosis of ASD was confirmed by expert clinicians, through clinical evaluation, medical reports and the Autism Diagnostic Observation Schedule-2 (ADOS-2) [52]. ASD group had a mean ADOS severity score of 6.2 \pm 2.43 out of 10 [52-54]. Full-scale IQ was measured using the two-subtest form of the Wechsler Abbreviated Scale of Intelligence (WASI) [55], except for 1 ASD and 2 control participants. The two groups were matched for IQ $(\mathrm{ASD}$ mean $=114.0 \pm 18$, controls mean $=114.3 \pm 10.1)$.

\section{Emotional face task in MEG}

The task used in the present study has been previously described [41]. Briefly, during each trial, a face (angry, happy or neutral) was shown on either side of a central fixation cross, for $80 \mathrm{~ms}$, to avoid time for any saccades; on the other side, a scrambled pattern of the face, matched for luminosity, contrast and colour, was presented simultaneously. A varying inter-trial interval (1300-1500 ms) preceded the next stimulus presentation. Participants were instructed to press left or right buttons as quickly as possible to indicate the side of the scrambled pattern, ignoring the faces. Thus, the processing of the emotional content remained implicit. The stimuli consisted of 75 colour photographs of 25 actors, posing for each of the three emotional expressions, taken from the NimStim Set of Facial expression [56]. Development of the MacBrain Face Stimulus Set was overseen by Nim Tottenham and supported by the John D. and Catherine T. MacArthur Foundation Research Network on Early Experience and Brain Development. Please contact Nim Tottenham at tott0006@tc.umn.edu for more information concerning the stimulus set. The emotional faces and their scrambled counterparts were presented in random order, twice on each side, resulting in a total of 300 trials. Response latencies were recorded. Images were back-projected onto a screen at $79 \mathrm{~cm}$ from the eyes, with a visual angle of $6.9^{\circ}$, thus in the parafoveal region.

\section{Neuroimaging data}

MEG signal was recorded inside a magnetically shielded room, with a 151-channel CTF system (CTF-MISL, Coquitlam, BC, Canada), at a $600-\mathrm{Hz}$ sampling rate. An online low-pass filter at $150 \mathrm{~Hz}$ was applied to the data, along with a third-order gradient cancellation. Fiducial coils on the left and right pre-auricular points and on the nasion of each subject allowed head position to be recorded continuously during the task. After the MEG, a T1-weighted MRI sequence was acquired (3D SAG MRPAGE, GRAPPA $=2, \mathrm{TR}=2300 \mathrm{~ms}, \mathrm{TE}=2.96 \mathrm{~ms}$, $\mathrm{FA}=9^{\circ}, 256 \times 256$ matrix, 192 slices, thickness $=1 \mathrm{~mm}$, isotropic voxels) with a 12-channel head coil in a $3 \mathrm{~T}$ MRI scanner (MAGNETOM Tim Trio, Siemens AG, Erlangen, Germany). The same fiducial points used in MEG were replaced with radio-opaque markers for the MRI, to allow for precise MEG/MRI co-registration. In both the MEG and MRI, the participants lay supine.

\section{Data processing and statistics Behavioural data}

Response latencies were entered in a mixed analysis of variance (ANOVA) with group (ASD/TD) as a between factor, and emotion (angry/happy/neutral) as a within factor. STATISTICA 7.0 software (StatSoft Inc, Tulsa, $\mathrm{OK})$ was used for statistical analysis.

\section{MEG data: preprocessing}

MEG data were band-pass filtered offline $(1-150 \mathrm{~Hz})$, and a notch filter at $60 \mathrm{~Hz}$ and harmonics was applied. Epochs were created in the broad -1500- to 3000-ms interval around stimulus presentation, in order to prevent boundary effects, and were excluded when intratrial head motion exceeded $5 \mathrm{~mm}$. The median head position from the remaining epochs was used for source reconstruction. A single shell head model was calculated from each individual's MRI and each individual's brain space was normalized to a template brain (ICBM 152) [57]. The time-series of brain activation for the 90 seeds of the Automated Anatomical Labeling (AAL) atlas [58] were estimated through a linearly constrained minimum variance (LCMV) beamformer [59]. Beamformers act as spatial filters, which reconstruct the signal from the desired region of interest (ROI) while suppressing signals from all other unwanted sources [60]. Due to the minimization of the contribution of the sources outside the ROI, beamformers are effective at suppressing ocular and non-ocular artefacts, therefore not requiring trial- 
by-trial artefact rejection based on visual inspection [61]. Moreover, as detailed below, the connectivity measure which was employed in the present study (the phase lag index; PLI) protects from potentially residual noise from common sources.

\section{MEG data: functional connectivity}

The time-series from each ROI were filtered into canonical bands (theta 4-7 Hz; alpha 8-14; beta 15-30; gamma 30-80), and a Hilbert transform was applied to the filtered data to extract the instantaneous phase at each time sample for each frequency. The PLI was calculated to estimate the cross-trial degree of phase synchronization between all pairwise combinations of the seeds for every time point, resulting in a $90 \times 90$ adjacency matrix for each subject, at each sample. The PLI quantifies the phase synchrony by calculating the asymmetry of the distribution of the instantaneous phase differences between two time series. It ranges from 0 (completely symmetric, or random, phase distribution) to 1 (completely asymmetric phase distribution); since field/spread volume conduction or leakage of common sources would determine a zero (or $180^{\circ}$ ) phase lag between the two sources, this measure is unaffected by spurious coupling that could be generated by artefacts instantaneously projecting to each of the ROIs.

PLI values from each participant's $90 \times 90$ matrix at each time point were averaged, to extract the time course of the mean "whole-brain" (entire seed grid) response in functional connectivity. The values were baselined $(-200-$ $0 \mathrm{~ms}$ ), and a grand average across groups and condition was computed. Beta and gamma connectivity peaked within $300 \mathrm{~ms}$, as expected considering their role in rapid brain response to emotional faces [42]. To determine the specificity of this effect, peaks in alpha and theta band connectivity were also computed and both resulted in peak connectivity after $400 \mathrm{~ms}$, thus, after the time of interest for the present study. Therefore, as the aim of the study was investigating functional connectivity responses during quick and automatized implicit processing of emotional stimuli $(<400 \mathrm{~ms})$, we selected beta and gamma bands for further analyses. To determine the time windows of interest for the statistical analyses, a data-driven approach was chosen. In order to focus on the main response, the time window of interest was defined as the full duration at half maximum-the temporal equivalent of FWHM-centred on the peak in PLI. Thus, for beta band we focused on the 227-337-ms interval and for gamma band on the 142-192-ms interval (Fig. 1). Based on these time windows, a temporally averaged adjacency matrix was generated separately for each frequency and emotion. The network-based statistics (NBS) [62] was used to compute statistical contrasts within groups (active window vs. baseline) and between groups (active windows,
ASD vs. TD). NBS considers the connectivity matrix in terms of graph theory, accounting for their topological distribution: first, a test statistic for each individual connection is computed (a $t$ test in the present study); the matrix is then thresholded and any connected structures (i.e. components) that might be present in the suprathreshold connections are identified. Thus, a component is defined as contiguous groups of network nodes bound by suprathreshold connections. A $p$ value corrected by Family Wise Error rate is assigned to each component, by means of permutation testing, as follows. For each permutation ( $n=5000$ in the present study), the group/condition to which each participant belongs is shuffled randomly and the corresponding maximal component size is calculated and stored. Thus, an empirical estimate of the null distribution of the maximal component size is generated. Finally, the number of permutations for which the maximal component size is greater than the original one, normalized for the total number of permutations, determines the $p$ value of the component.

The threshold for the $t$-statistic for the initial univariate testing was adapted to the data, as suggested by Zalesky and colleagues [62]. Therefore, for within contrasts, thresholding values were set to 7.5 and 5 for beta and gamma bands, respectively. For between contrasts, a value of 3.1 was used for both bands. The importance of each node was assessed using the node strength measure (i.e. the sum of weights of links connected to the node), computed using the Brain Connectivity Toolbox [63]. Results were considered to be statistically significant at $p<0.05$, corrected. Both the significant components and the node strength data were visualized using BrainNet Viewer [64].

\section{MEG data: time-frequency analyses}

To complement the whole-brain connectivity findings, event-related synchronization/de-synchronization (ERS/ D) were explored in specific seeds of interest: seeds of interest were selected among network nodes involved in the significant component that emerged from betweengroups statistical contrasts with NBS. In particular, seed selection was based on the literature of brain regions involved in processing of emotional faces in both TD and ASD individuals. A Morlet wavelet transformation, as implemented in Fieldtrip [65], on each selected seed's estimated time-series was applied; the proportion of change relative to the baseline ( -200 to $0 \mathrm{~ms}$ ) in each frequency bin at each 10-ms time point was computed.

\section{Results}

\section{Response latency}

Neither the main effects for group and emotion, nor the group $\times$ emotion interaction were significant for the response latency (all $p>0.21$ ), indicating that both groups performed equally well on the task. 


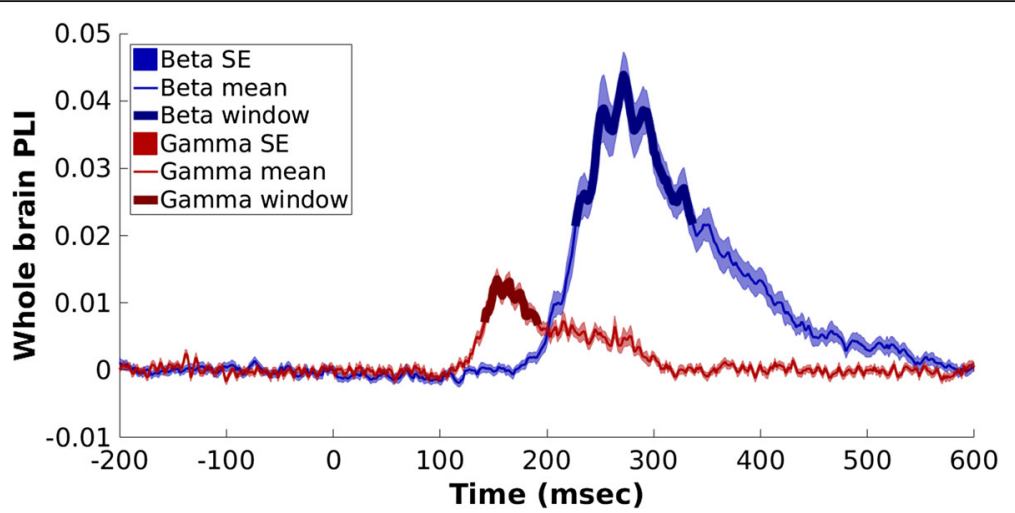

Fig. 1 Mean "whole brain" connectivity response in beta and gamma bands across groups and conditions. The time windows selected for further analyses are represented with thicker lines

\section{Connectivity: within groups}

\section{Beta band}

Both controls and ASD participants displayed a significant increase in beta connectivity in the active window compared to baseline, for each emotion condition (Fig. 2, left; Additional file 1: Table S1). Across groups and conditions, occipital areas involved in basic visual processing (e.g. calcarine sulcus, cuneus) were among the most connected seeds (Additional file 2: Table S2). Both groups showed also an involvement of areas related to the core face processing system $[50,66]$, such as the inferior occipital and the lingual gyri. The increased connectivity also included regions of the emotional system for face processing (amygdala, insula, and striatum (i.e. pallidum, caudate and putamen in the AAL)). The face-related extended system in the model from Gobbini and Haxby includes both the emotional system and other brain areas which participate in retrieval of

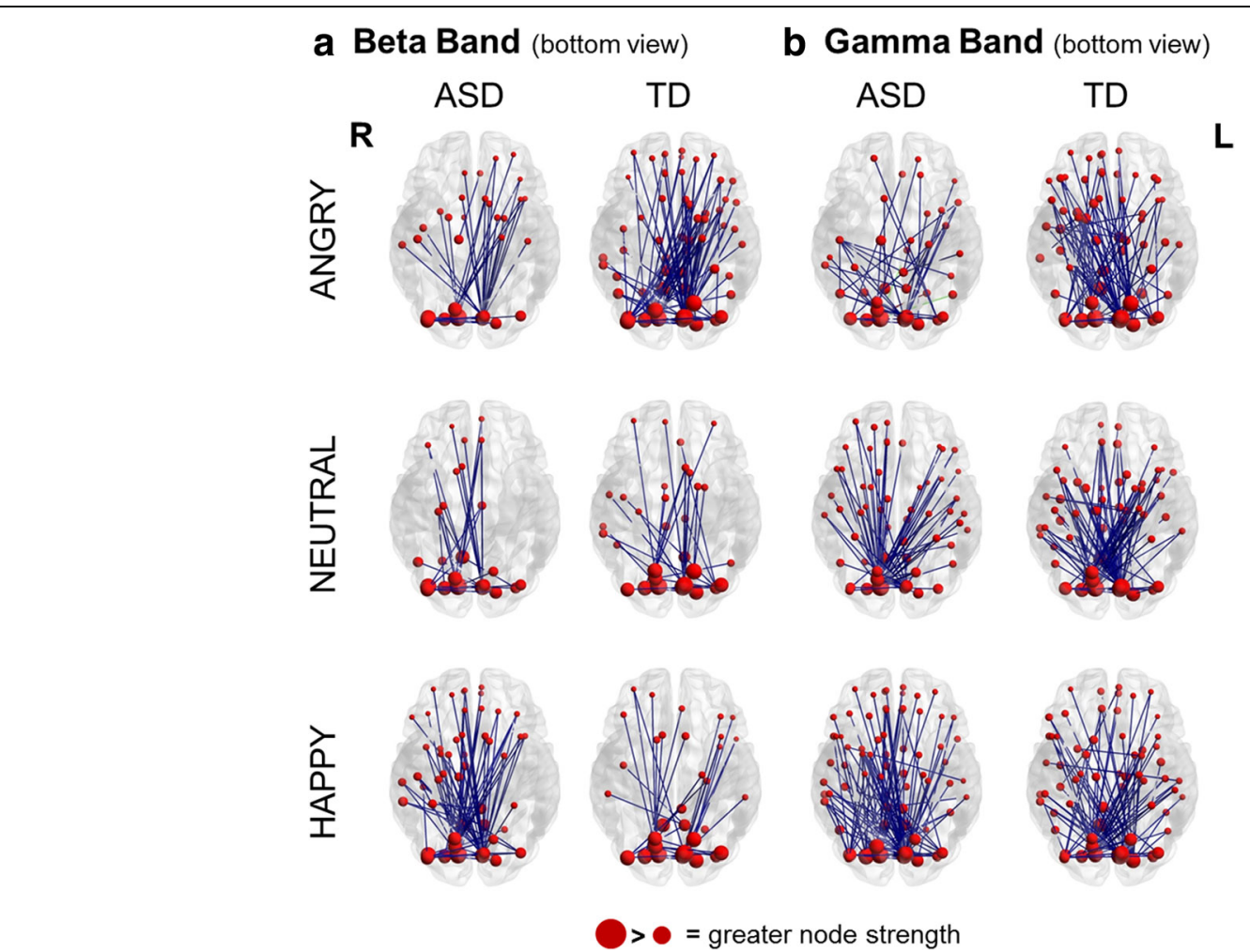

Fig. 2 Task-dependent increases in connectivity for each group and condition. a Task-dependent increases in beta band; $\mathbf{b}$ task-dependent increases in gamma band. Blue lines represent significant connections between seeds. The brains are viewed from the bottom. $R=$ right, $L=l$ left, $A S D=$ autism group, TD = typically developed group 
different aspects of person knowledge (i.e. anterior and posterior cingulate, precuneus, posterior superior temporal sulcus/temporo-parietal junction, and anterior temporal cortices) [50], which were also involved in increased beta connectivity.

\section{Gamma band}

Control and ASD participants also displayed a significant increase in gamma connectivity in the active window compared to baseline, for each condition (Fig. 2, right; Additional file 1: Table S1). For the gamma band, the calcarine sulcus and the cuneus were highly connected, as well as the nodes of the core, extended and, in particular, emotion systems for face processing. Of note, in contrast to the beta band results, the fusiform gyri, and in particular the right fusiform, were almost always present among the nodes involved in increased gamma connectivity across groups and conditions (Additional file 3: Table S3).

\section{Connectivity: between groups}

Beta band

From the contrasts between groups, a difference emerged for the processing of angry faces. Specifically, participants with ASD showed reduced beta interregional phase-locking compared to controls $(p=0.04$, corrected). Reduced beta connectivity involved mainly connections of frontal and limbic areas with the calcarine sulcus and the lingual gyrus in the occipital lobe (Fig. 3, top; Table 1). The reduction in connectivity in ASD emerged for all the areas of the emotion system for face processing, within the left hemisphere (Fig. 3, bottom). Overall, the network nodes involved were largely left-lateralized, apart from a few frontal and parietal sites. No significant results emerged for the between-group contrasts in the happy or neutral condition.

\section{Gamma band}

None of the contrasts reached statistical significance.

\section{BETA BAND: ANGRY (ASD < TD)}

a Left view

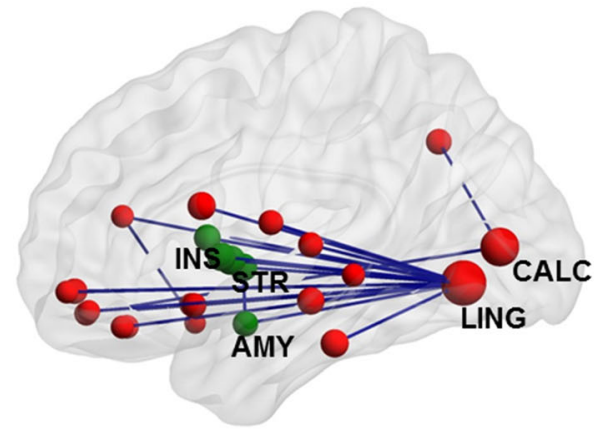

b Bottom view

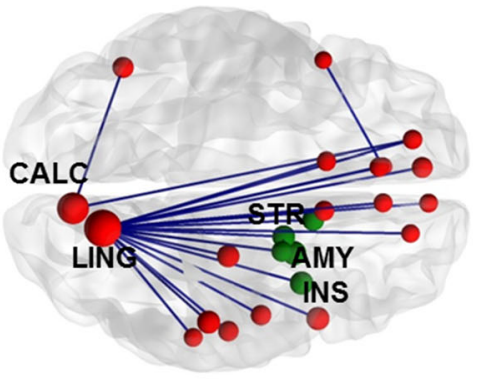

\section{Emotional system for face processing}
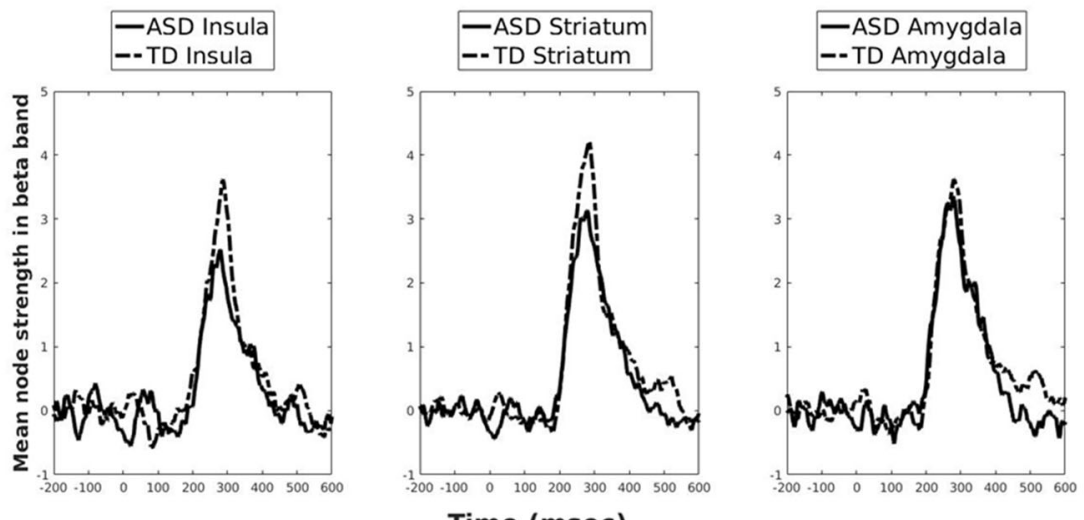

Time (msec)

Fig. 3 Between-group comparison of phase synchronization in the beta band, during processing of angry faces. a Left and $\mathbf{b}$ bottom view of reduced beta band interregional phase-locking in ASD individuals compared to TD controls. In green, seeds from the emotional system for face processing. Bottom time course of node strength of the seeds within the emotional system (smoothed for clarity with a 30-ms moving average) 
Table 1 Beta hypo-connectivity in ASD compared to TD individuals: involved areas, number of connected seeds and group difference in node strength (TD-ASD)

\begin{tabular}{llll}
\hline Area & Hemisphere & $\begin{array}{l}\text { Number of } \\
\text { connections }\end{array}$ & $\begin{array}{l}\Delta \text { Node strength } \\
\text { (TD-ASD) }\end{array}$ \\
\hline Lingual & $(\mathrm{L} / \mathrm{R})$ & 20 & 2.74 \\
Calcarine & $\mathrm{L}$ & 2 & 1.89 \\
Pallidum & $\mathrm{L}$ & 2 & 0.50 \\
Amygdala & $\mathrm{L}$ & 2 & 0.22 \\
Superior orbitofrontal cortex & $\mathrm{R}$ & 2 & 0.02 \\
Anterior cingulate cortex & $\mathrm{R}$ & 2 & -0.11 \\
Putamen & $\mathrm{L}$ & 1 & 0.72 \\
Insula & $\mathrm{L}$ & 1 & 0.61 \\
Frontal inferior operulum & $\mathrm{L}$ & 1 & 0.57 \\
Inferior temporal gyrus & $\mathrm{L}$ & 1 & 0.55 \\
Hippocampus & $\mathrm{L}$ & 1 & 0.47 \\
Caudate & $\mathrm{L}$ & 1 & 0.39 \\
Angular gyrus & $\mathrm{L}$ & 1 & 0.26 \\
Superior temporal gyrus & $\mathrm{L}$ & 1 & 0.16 \\
Rolandic operculum & $\mathrm{L}$ & 1 & 0.16 \\
Olfactory cortex & $\mathrm{L}$ & 1 & 0.11 \\
Gyrus rectus & $\mathrm{R}$ & 1 & 0.00 \\
Olfactory cortex & $\mathrm{R}$ & 1 & -0.02 \\
Gyrus rectus & $\mathrm{L}$ & 1 & -0.07 \\
Middle temporal gyrus & $\mathrm{L}$ & 1 & -0.08 \\
Superior orbitofrontal cortex & $\mathrm{L}$ & 1 & -0.14 \\
Medial orbitofrontal cortex & $\mathrm{L}$ & 1 & -0.19 \\
Superior temporal pole & $\mathrm{R}$ & 1 & -0.27 \\
Medial orbitofrontal cortex & $\mathrm{R}$ & 1 & -0.33 \\
\hline & & & \\
& & 1 & 1 \\
\hline
\end{tabular}

\section{Time-frequency responses}

Since a pattern of reduced connectivity emerged in the ASD group in the beta band, we investigated the timefrequency responses in the beta band in specific seeds of interest, as defined in the "Methods" section. Based on the large literature on TD and ASD individuals and the importance of amygdalae and insulae in processing of emotional faces [51], we focused on these seeds. Moreover, the majority of the connections involved the lingual gyrus, which, due to its importance in face processing [66], was also included for further investigation. All the selected sites were left-lateralized, in line with the between-group connectivity results.

Across seeds there was a general pattern of desynchronization in the beta band overlapping in time with the peak in the connectivity response (Fig. 4; Additional file 4: Figure S1). Thus, we averaged beta ERD values in time, between 220 and $340 \mathrm{~ms}$, and in frequency, between 18 and $30 \mathrm{~Hz}$, on each selected seed. Mean values were entered in a mixed ANOVA with group as a between-group factor, and emotion as a within-group factor. A significant effect for group emerged in the amygdala $\left(F_{(1,42)}=9.68, p=0.003\right)$ indicating that ERD was reduced in ASD compared to TD irrespective of stimuli's category. No other main effects/ interactions emerged for amygdala, insula nor lingual seeds (all $p>0.09$ ).

\section{Discussion}

To the best of our knowledge, this is the first study investigating whole-brain MEG connectivity in adults with ASD compared to TD controls during implicit processing of emotional faces. The ASD group showed reduced connectivity in the beta band compared to TD subjects during implicit processing of angry faces. In particular, reduced connectivity in ASD involved several areas of the social brain. Specifically, both low-level visual areas, such as the calcarine cortex, and face-sensitive regions, such as the lingual gyrus and the inferior temporal gyrus [66], were significantly hypo-connected in ASD compared to TD individuals. Other regions pertaining to the extended system for face processing [50], such as the superior temporal gyrus, the temporal pole and the anterior cingulate cortex, were also under-connected in ASD. Importantly, the network included the amygdala, the insula and the striatum, which are central in the emotional processing of faces. Of note, these structures are deep and/or subcortical regions of the brain; therefore, the present results align with the large body of MEG literature which supports the use of beamforming algorithms for MEG source reconstruction of subcortical neural activity [41, 46, 67-75]. Based on beamforming solutions, functional connectivity within thalamo-cortico-limbic circuits has been studied using MEG during processing of emotional faces [48]. Moreover, our results largely converge with recent fMRI findings which reported reduced connectivity between limbic (e.g. amygdala) and cortical regions of the social brain in ASD compared to TD individuals, during both explicit [27] and implicit [28] processing of emotional faces. The present study adds to the literature by providing a precise characterization in the time domain of reduced functional connectivity in ASD individuals.

The majority of the seeds involved were lateralized to the left hemisphere. A model has been proposed that anterior regions of the left cerebral hemisphere subtend approach motivation, while the anterior regions of the right hemisphere are responsible for withdrawal motivation [76-82]. It has been shown that, although a negatively valenced emotion, anger elicits approach motivation and behaviours, associated with greater left than right brain activation [83-87]. Thus, hypo-connectivity in the left hemisphere during processing of angry faces supports the theory of poor motivation toward social 


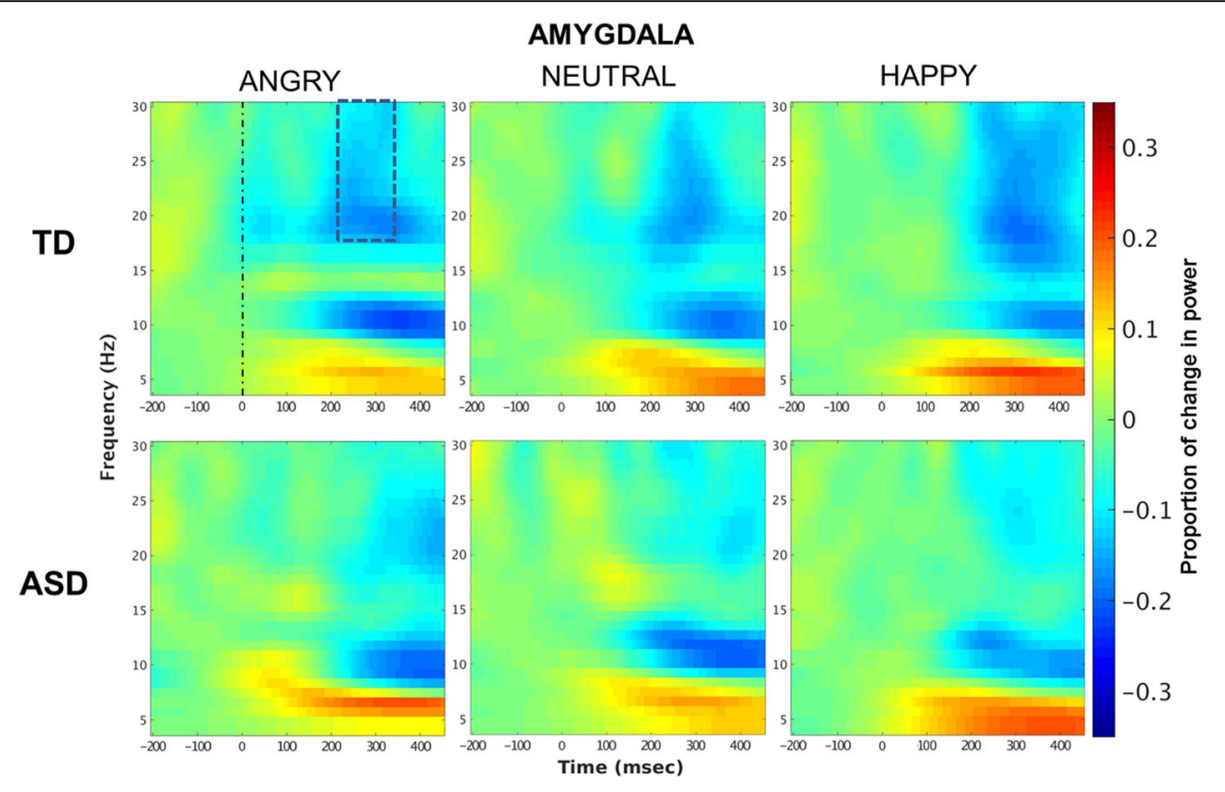

Fig. 4 Left amygdala: time-frequency activation. Beta de-synchronization occurred in a time window overlapping with the connectivity peak (blue scattered line). Beta ERD in the amygdala was smaller (less negative) in ASD compared to TD across stimuli ( $p=0.003$ )

stimuli in ASD compared to TD individuals [1-3]. Intriguingly, our findings largely overlap with results from Leung and colleagues who used the same task on adolescents with autism (range 12-15 years) [41]. Nonetheless, the latter study did not find the strong left lateralization for angry faces. This suggests that hemispheric specialization in emotional face processing evolves during the development and ASD individuals may show an atypical developmental trajectory. Supporting this interpretation, subjects with ASD show an opposite pattern of MEG lateralization compared to TD individuals for other cognitive functions, such as language. While TD individuals showed a shift toward left-lateralization for language as age increased, those with ASD showed the opposite pattern, that is a right-sided lateralization with age [88]. This is in line with structural studies which highlight a shift toward right lateralization in cortical volume in ASD [89]. Interpretations of laterality effects, however, ought to be taken with caution, since no statistical tests on connectivity strength at left and right sites were run. Beamformers are not ideal for estimation of stimulus-driven oscillatory activity of bilateral sources, which can be highly synchronized [90], and therefore for testing laterality effects. The supposition that differences exist in the developmental trajectory of emotional processing should be investigated using other source localization algorithms as well, and by research comparing connectivity asymmetries at different ages, or longitudinally.

Consistent with other research, under-connectivity in social brain areas in ASD emerged for angry, but not happy faces [41, 49]. Behavioural studies have shown that autism is associated with specific difficulties in processing of negative emotional faces [91], and in particular angry faces [92]. In a previous study on adolescents with ASD [41], hypo-connectivity for angry faces had been related to the observation that the ability to identify negative emotions, such as anger and fear, matures at later ages, and therefore negative emotions may require more complex processing compared to positive emotions [93-96]. This development-related explanation may not hold for our results with adults with ASD. Another possible interpretation relates to angry faces being negatively valenced social stimuli, which convey information about potential danger or harm for the individual. Thus, compared to positively valenced stimuli, they are thought to be processed very quickly in the brain, allowing the organism to prepare a rapid behavioural response [42, 78, 97]. Therefore, it is plausible that atypical functional connectivity in ASD emerged due to deficits in the high-demanding quick processing requirements of negative content, especially in the context of our very rapidly presented implicit processing task.

The present results also extend previous EEG/MEG studies showing the importance of beta band in processing of emotional valence of social and non-social emotional stimuli $[35,42,98]$. In particular, we replicated the increase in signal coherence within the beta band reported during processing of visual emotional stimuli $[39,41]$. Moreover, by combining the connectivity analysis with a time-frequency analysis on seeds of interests, it emerged that the increase in beta connectivity overlapped in time with a decrease in beta power in the 220-340-ms window. Our results align with studies 
showing that event-related beta power correlates negatively with the hemodynamic response in task-relevant brain areas [99, 100]. Beta de-synchronization has been reported during processing of salient external events [101], both in EEG [43, 102] and MEG studies [103], and it has been interpreted as an adaptation process in response to the perceived emotional information [43]. In the present study, beta ERD in the left amygdala was reduced in ASD compared to TD individuals irrespective of stimulus condition, suggesting that participants with ASD had a less pronounced response to stimulus salience. Overall, we propose that the general difficulty in extracting emotional information from faces in ASD, as indicated by amygdala hypo-responsivity, is associated with impaired long-range communication between areas of the social brain, which instead is specific for highly salient angry faces.

It was surprising that the involvement of the fusiform gyri did not emerge in the connectivity differences between ASD and TD individuals. This may be attributed to the timing of the beta peak in connectivity, which occurred after the time window where the fusiform activation is usually maximal (i.e. 130-200 ms) [104]. As a confirmation of this hypothesis, the within group results showed that the fusiform was more involved in the gamma response to the task, which peaked between 142 and $192 \mathrm{~ms}$. Previous research demonstrated that, during processing of human faces, gamma band is associated with "structural encoding", which represents the integration of the configural metrics and face-feature characteristics into a detailed representation [105]. Other MEG studies also found that face-induced gamma oscillations, albeit not representing the same neurophysiological process as the M170, were generated by common sources, including the right fusiform gyrus [106]. Although connectivity in gamma band in autism during processing of emotional faces has been rarely investigated, one study reported differences in gamma power during emotional face processing in ASD compared to TD individuals [103]. Thus, while the group effect in the gamma band connectivity was not significant in the present study, future studies should further clarify the role of gamma band in emotional face processing in ASD.

\section{Conclusions}

The present study provides the first demonstration of hypo-connectivity in adults with ASD compared to TD individuals during emotional face processing, using a whole-brain MEG approach. Under-connectivity in ASD was evident in the beta band during processing of angry faces and involved key areas of the social brain largely in the left hemisphere, such as the amygdala, the insula and the lingual gyrus. Complementary time-frequency analyses highlighted hypo-activation of the amygdala which, together with the pattern of under-connectivity in the social brain, could contribute to the socioemotional difficulties in ASD. These findings shed light on important neural underpinnings of the disorder, providing potential for the development of new techniques to aid in the diagnosis and assessment of the efficacy of treatments.

\section{Additional files}

\begin{abstract}
Additional file 1: Table S1. Significant NBS components for beta and gamma bands in ASD and TD group (active window vs. baseline). (DOCX $15 \mathrm{~kb}$ )

Additional file 2: Table S2. Within connectivity in the beta band: Number of connections for each AAL node within the significant NBS components. Nodes are ordered by the sum of the connections across groups and conditions (from the greater to the smaller value). (DOCX $18 \mathrm{~kb}$ )
\end{abstract}

Additional file 3: Table S3. Within connectivity in the gamma band: Number of connections for each AAL node within the significant NBS components. Nodes are ordered by the sum of the connections across groups and conditions (from the greater to the smaller value). (DOCX $22 \mathrm{~kb}$ )

Additional file 4: Figure S1. Time-frequency activation: Beta de-synchronization in the left lingual gyrus and insula. (TIF 1151 kb)

\section{Abbreviations}

AAL: Automated Anatomical Labeling; ADOS-2: Autism Diagnostic Observation Schedule-2; ANOVA: Analysis of variance; ASD: Autism spectrum disorder; EEG: Electroencephalography; ERS/D: Event-related synchronization/ de-synchronization; fMRI: Functional magnetic resonance imaging; FWHM: Full-width at half maximum; IQ: Intelligence quotient; LCMV: Linearly constrained minimum variance; MEG: Magnetoencephalography;

MRI: Magnetic resonance; NBS: Network-based statistics; PLI: Phase lag index; ROI: Region of interest; TD: Typically developed; WASI: Wechsler Abbreviated Scale of Intelligence

\section{Acknowledgements}

The authors would like to thank Marc Lalancette for help in data collection, as well as Anne Keller and Simeon Wong for support in the data analysis.

The authors acknowledge Klara Kovarski for reference suggestions.

\section{Funding}

This work was funded by the Canadian Institutes of Health Research (CIHR) (Grants: MOP-119541 and MOP-142379) to MJT. The funder had no role in the design and conduct of the study; collection, management, analysis, and interpretation of the data; and preparation, review, or approval of the manuscript.

\section{Availability of data and materials}

The datasets during and/or analysed during the current study available from the corresponding author on reasonable request.

\section{Authors' contributions}

RM participated in the data analysis, result interpretation, and drafting of the manuscript. RCL participated in the design of the study, acquisition of data and editing of the manuscript. MJT participated in the conception and design of the study, guiding data analysis, result interpretation and editing of the manuscript. BTD participated in the creation of the data analysis pipeline, guiding data analyses, result interpretation, and editing of the manuscript. All authors read and approved the final manuscript.

Competing interests

The authors declare that they have no competing interests. 


\section{Consent for publication}

Not applicable.

\section{Ethics approval and consent to participate}

All participants read and signed an informed written consent, after receiving a complete description of the study. Testing and recordings were completed in the MEG Lab at the Hospital for Sick Children, and the Research Ethics Board of the hospital gave institutional approval for the study.

\section{Author details}

'Department of Diagnostic Imaging, Hospital for Sick Children, 555 University Avenue, Toronto, Ontario M5G 1X8, Canada. ${ }^{2}$ Department of General Psychology, University of Padova, Via Venezia 8, 35131 Padova, Italy. ${ }^{3}$ Neurosciences \& Mental Health, Hospital for Sick Children Research Institute, 555 University Avenue, Toronto, Ontario M5G 1X8, Canada. ${ }^{4}$ Department of Medical Imaging, Faculty of Medicine, University of Toronto, 263 McCaul Street - 4th Floor, Toronto, Ontario M5T 1W7, Canada. ${ }^{5}$ Department of Psychology, University of Toronto, 100 St. George Street, 4th Floor, Sidney Smith Hall, Toronto, Ontario M5S 3G3, Canada.

\section{Received: 20 July 2016 Accepted: 16 February 2017} Published online: 22 February 2017

\section{References}

1. Schultz RT. Developmental deficits in social perception in autism: the role of the amygdala and fusiform face area. Int J Dev Neurosci. 2005;23(2-3):125-41.

2. Chevallier C, Kohls G, Troiani V, Brodkin ES, Schultz RT. The social motivation theory of autism. Trends Cogn Neurosci. 2013;16(4):231-9.

3. Mundy P, Newell L. Attention, joint attention, and social cognition. Curr Dir Psychol Sci. 2007;16(5):269-74.

4. Baron-Cohen S, Leslie AM, Frith U. Does the autistic child have a "theory of mind" ? Cognition. 1985;21(1):37-46.

5. Gaigg SB. The interplay between emotion and cognition in autism spectrum disorder: implications for developmental theory. Front Integr Neurosci. 2012;6:113.

6. Adolphs R, Sears L, Piven J. Abnormal processing of social information from faces in autism. J Cogn Neurosci. 2001;13(2):232-40.

7. Baron-Cohen S, Ring HA, Bullmore ET, Wheelwright S, Ashwin C, Williams SCR. The amygdala theory of autism. Neurosci Biobehav Rev. 2000;24(3):355-64

8. Di Martino A, Ross K, Uddin LQ, Sklar AB, Castellanos FX, Milham MP. Functional brain correlates of social and nonsocial processes in autism spectrum disorders: an activation likelihood estimation meta-analysis. Biol Psychiatry. 2009;65(1):63-74.

9. Critchley HD. The functional neuroanatomy of social behaviour: changes in cerebral blood flow when people with autistic disorder process facial expressions. Brain. 2000;123(11):2203-12.

10. Pelphrey KA, Carter EJ. Brain mechanisms for social perception. Ann N Y Acad Sci. 2008;1145(1):283-99.

11. Just MA, Keller TA, Malave VL, Kana RK, Varma S. Autism as a neural systems disorder: a theory of frontal-posterior underconnectivity. Neurosci Biobehav Rev. 2012;36(4):1292-313.

12. Maximo JO, Cadena EJ, Kana RK. The implications of brain connectivity in the neuropsychology of autism. Neuropsychol Rev. 2014;24(1):16-31.

13. Ramachandran VS, Oberman LM. Broken mirrors: a theory of autism. Sci Am. 2006;295(5):62-9.

14. Uddin LQ, Menon V. The anterior insula in autism: under-connected and under-examined. Neurosci Biobehav Rev. 2009;33(8):1198-203.

15. Frith CD, Frith U. Social cognition in humans. Curr Biol. 2007:17(16):724-32

16. Frith CD. The social brain? Philos Trans R Soc B Biol Sci. 2007;362(1480):671-8.

17. Pessoa L, Adolphs R. Emotion processing and the amygdala: from a "low road" to "many roads" of evaluating biological significance. Nat Rev Neurosci Nat Publ Group. 2010;11(11):773-83.

18. Fanselow MS, Dong H-W. Are the dorsal and ventral hippocampus functionally distinct structures? Neuron. 2010;65(1):7-19.

19. Chen Y-H, Dammers J, Boers F, Leiberg S, Edgar JC, Roberts TPL, et al. The temporal dynamics of insula activity to disgust and happy facial expressions: a magnetoencephalography study. Neuroimage. 2009;47(4):1921-8.

20. Olson IR, McCoy D, Klobusicky E, Ross LA. Social cognition and the anterior temporal lobes: a review and theoretical framework. Soc Cogn Affect Neurosci. 2013;8(2):123-33.
21. Deen B, McCarthy G. Reading about the actions of others: biological motion imagery and action congruency influence brain activity. Neuropsychologia. 2010;48(6):1607-15.

22. Hamilton AF de C, Grafton ST. Goal representation in human anterior intraparietal sulcus. J Neurosci. 2006;26(4):1133-7.

23. Turken AU, Dronkers NF. The neural architecture of the language comprehension network: converging evidence from lesion and connectivity analyses. Front Syst Neurosci. 2011;5:1

24. Gotts SJ, Simmons WK, Milbury LA, Wallace GL, Cox RW, Martin A. Fractionation of social brain circuits in autism spectrum disorders. Brain. 2012;135(9):2711-25.

25. Ebisch SJH, Gallese V, Willems RM, Mantini D, Groen WB, Romani GL, et al. Altered intrinsic functional connectivity of anterior and posterior insula regions in high-functioning participants with autism spectrum disorder. Hum Brain Mapp. 2011:32(7):1013-28.

26. Kleinhans NM, Richards T, Sterling L, Stegbauer KC, Mahurin R, Johnson LC, et al. Abnormal functional connectivity in autism spectrum disorders during face processing. Brain. 2008;131(4):1000-12.

27. Wicker B, Fonlupt P, Hubert B, Tardif C, Gepner B, Deruelle C. Abnormal cerebral effective connectivity during explicit emotional processing in adults with autism spectrum disorder. Soc Cogn Affect Neurosci. 2008:3(2):135-43.

28. Kana RK, Patriquin MA, Black BS, Channell MM, Wicker B. Altered medial frontal and superior temporal response to implicit processing of emotions in autism. Autism Res. 2016;9(1):55-66.

29. Batty M, Meaux E, Wittemeyer K, Rogé B, Taylor MJ. Early processing of emotional faces in children with autism: an event-related potential study. J Exp Child Psychol. 2011;109(4):430-44.

30. Batty M, Taylor MMJ. Early processing of the six basic facial emotional expressions. Cogn Brain Res. 2003;17(3):613-20.

31. Batty M, Taylor MJ. The development of emotional face processing during childhood. Dev Sci. 2006;9(2):207-20.

32. Cohen D, Cuffin BN. A method for combining MEG and EEG to determine the sources. Phys Med Biol. 1987;32(1):85.

33. Hari R. Magnetoencephalography: methods and applications. In: Niedermeyer's Electroencephalography. Philadelphia: Lippincott Williams \& Wilkins; 2011. p. 865-900.

34. Garcia-Garcia M, Yordanova J, Kolev V, Domínguez-Borràs J, Escera C. Tuning the brain for novelty detection under emotional threat: the role of increasing gamma phase-synchronization. Neuroimage. 2010;49(1):1038-44.

35. Güntekin B, Başar E. Event-related beta oscillations are affected by emotional eliciting stimuli. Neurosci Lett. 2010;483(3):173-8.

36. Keil A, Müller MM, Gruber T, Wienbruch C, Stolarova M, Elbert T. Effects of emotional arousal in the cerebral hemispheres: a study of oscillatory brain activity and event-related potentials. Clin Neurophysiol. 2001;112(11):2057-68

37. Keil A, Stolarova M, Moratti S, Ray WJ. Adaptation in human visual cortex as a mechanism for rapid discrimination of aversive stimuli. Neuroimage. 2007;36(2):472-9.

38. Martini N, Menicucci D, Sebastiani L, Bedini R, Pingitore A, Vanello N, et al. The dynamics of EEG gamma responses to unpleasant visual stimuli: from local activity to functional connectivity. Neuroimage. 2012;60(2):922-32

39. Miskovic V, Schmidt LA. Cross-regional cortical synchronization during affective image viewing. Brain Res. 2010;1362:102-11.

40. Müller MM, Keil A, Gruber T, Elbert T. Processing of affective pictures modulates right-hemispheric gamma band EEG activity. Clin Neurophysiol. 1999;110(11):1913-20.

41. Leung RC, Ye AX, Wong SM, Taylor MJ, Doesburg SM. Reduced beta connectivity during emotional face processing in adolescents with autism. Mol Autism. 2014;5(1):51.

42. Güntekin $B$, Başar $E$. A review of brain oscillations in perception of faces and emotional pictures. Neuropsychologia. 2014;58(1):33-51.

43. Jessen S, Kotz SA. The temporal dynamics of processing emotions from vocal, facial, and bodily expressions. Neuroimage. 2011;58(2):665-74.

44. Jabbi M, Kohn PD, Nash T, lanni A, Coutlee C, Holroyd T, et al. Convergent BOLD and beta-band activity in superior temporal sulcus and frontolimbic circuitry underpins human emotion cognition. Cereb Cortex. 2015;25(7):1878-88.

45. Sato W, Kochiyama T, Uono S, Matsuda K, Usui K, Inoue Y, et al. Rapid amygdala gamma oscillations in response to fearful facial expressions. Neuropsychologia. 2011;49(4):612-7. 
46. Luo Q, Holroyd T, Jones M, Hendler T, Blair J. Neural dynamics for facial threat processing as revealed by gamma band synchronization using MEG. Neuroimage. 2007;34(2):839-47.

47. Luo Q, Mitchell D, Cheng X, Mondillo K, McCaffrey D, Holroyd T, et al. Visual awareness, emotion, and gamma band synchronization. Cereb Cortex. 2009; 19(8):1896-904.

48. Liu T-Y, Chen Y-S, Hsieh J-C, Chen L-F. Asymmetric engagement of amygdala and its gamma connectivity in early emotional face processing. PLoS One. 2015;10(1):1-16

49. Khan S, Gramfort A, Shetty NR, Kitzbichler MG, Ganesan S, Moran JM, et al. Local and long-range functional connectivity is reduced in concert in autism spectrum disorders. Proc Natl Acad Sci. 2013;110(8):3107-12.

50. Gobbini MI, Haxby JV. Neural systems for recognition of familiar faces. Neuropsychologia. 2007;45(1):32-41.

51. Nomi JS, Uddin LQ. Face processing in autism spectrum disorders: from brain regions to brain networks. Neuropsychologia. 2015;71:201-16.

52. Rutter M, DiLavore PC, Risi S, Gotham K, Bishop S. Autism diagnostic observation schedule: ADOS-2. Los Angeles: Western Psychological Services; 2012.

53. Hus V, Lord C. The Autism Diagnostic Observation Schedule, Module 4: Revised Algorithm and Standardized Severity Scores. J Autism Dev Disord. 2014;44(8):1996-2012.

54. Gotham K, Pickles A, Lord C. Standardizing ADOS Scores for a Measure of Severity in Autism Spectrum Disorders. J Autism Dev Disord. 2009;39(5):693-705.

55. Wechsler D. Wechsler abbreviated scale of intelligence. Psychological Corporation. 1999

56. Tottenham N, Tanaka JW, Leon AC, McCarry T, Nurse M, Hare TA, et al. The NimStim set of facial expressions: judgements from untrained research participants. Psychiatry Res. 2009;168(3):242-9.

57. Fonov VS, Evans AC, McKinstry RC, Almli CR, Collins DL. Unbiased nonlinear average age-appropriate brain templates from birth to adulthood. Neuroimage. 2009;47(1):S102.

58. Tzourio-Mazoyer N, Landeau B, Papathanassiou D, Crivello F, Etard O, Delcroix $\mathrm{N}$, et al. Automated anatomical labeling of activations in SPM using a macroscopic anatomical parcellation of the MNI MRI single-subject brain Neuroimage. 2002;15(1):273-89.

59. Van Veen B, van Drongelen W, Yuchtman M, Suzuki A. Localization of brain electrical activity via linearly constrained minimum variance spatial filtering. IEEE Trans Biomed Eng. 1997:44(9):867-80

60. Quraan MA, Cheyne D. Reconstruction of correlated brain activity with adaptive spatial filters in MEG. Neuroimage. 2010;49(3):2387-400.

61. Muthukumaraswamy SD. High-frequency brain activity and muscle artifacts in MEG/EEG: a review and recommendations. Front Hum Neurosci. 2013;7:138.

62. Zalesky A, Fornito A, Bullmore ET. Network-based statistic: identifying differences in brain networks. Neuroimage. 2010;53(4):1197-207.

63. Rubinov M, Sporns O. Complex network measures of brain connectivity: uses and interpretations. Neuroimage. 2010;52(3):1059-69.

64. Xia M, Wang J, He Y. BrainNet Viewer: a network visualization tool for human brain connectomics. PLoS One. 2013;8(7):e68910.

65. Oostenveld R, Fries P, Maris E, Schoffelen J-M. FieldTrip: open source software for advanced analysis of MEG, EEG, and invasive electrophysiological data. Comput Intell Neurosci. 2011;2011:1-9.

66. Fusar-Poli P, Placentino A, Carletti F, Landi P, Allen P, Surguladze S, et al. Functional atlas of emotional faces processing: a voxel-based meta-analysis of 105 functional magnetic resonance imaging studies. J Psychiatry Neurosci. 2009;34(6):418-32.

67. Luo Q, Cheng X, Holroyd T, Xu D, Carver F, Blair RJ. Theta band activity in response to emotional expressions and its relationship with gamma band activity as revealed by MEG and advanced beamformer source imaging. Front Hum Neurosci. 2013;7(February):940.

68. Maratos FA, Mogg K, Bradley BP, Rippon G, Senior C. Coarse threat images reveal theta oscillations in the amygdala: a magnetoencephalography study. Cogn Affect Behav Neurosci. 2009;9(2):133-43.

69. Cornwell BR, Carver FW, Coppola R, Johnson L, Alvarez R, Grillon C. Evoked amygdala responses to negative faces revealed by adaptive MEG beamformers. Brain Res. 2008;1244(November):103-12.

70. Garolera M, Coppola R, Muñoz KE, Elvevåg B, Carver FW, Weinberger DR, et al. Amygdala activation in affective priming: a magnetoencephalogram study. Neuroreport. 2007;18(14):1449-53.

71. Hung Y, Smith M L, Bayle DJ, Mills T, Cheyne D, Taylor MJ. Unattended emotional faces elicit early lateralized amygdala-frontal and fusiform activations. Neuroimage. 2010;50(2):727-33.
72. Quraan MA, Moses SN, Hung Y, Mills T, Taylor MJ. Detection and localization of hippocampal activity using beamformers with MEG: a detailed investigation using simulations and empirical data. Hum Brain Mapp. 2011;32(5):812-27.

73. Dunkley BT, Doesburg SM, Sedge PA, Grodecki RJ, Shek PN, Pang EW, et al. Resting-state hippocampal connectivity correlates with symptom severity in post-traumatic stress disorder. Neurolmage Clin. 2014;5:377-84.

74. Hung Y, Smith M L, Taylor MJ. Functional dissociations in prefrontalhippocampal working memory systems. Cortex. 2013;49(4):961-7.

75. Styliadis C, loannides AA, Bamidis PD, Papadelis C. Amygdala responses to valence and its interaction by arousal revealed by MEG. Int J Psychophysiol. 2014;93(1):121-33.

76. Konorski J. Integrative activity of the brain. An interdisciplinary approach. Chicago: University of Chicago Press; 1967.

77. Dickinson A, Pearce JM. Inhibitory interactions between appetitive and aversive stimuli. Psychol Bull. 1977;84(4):690-711

78. Lang PJ, Bradley MM, Cuthbert BN. Emotion, motivation, and anxiety: brain mechanisms and psychophysiology. Biol Psychiatry. 1998;44(12):1248-63.

79. Lang PJ, Bradley MM, Cuthbert BN. Emotion, attention, and the startle reflex. Psychol Rev. 1990;97(3):377-95.

80. Tomarken AJ, Davidson RJ, Wheeler RE, Doss RC. Individual differences in anterior brain asymmetry and fundamental dimensions of emotion. J Pers Soc Psychol. 1992;62(4):676-87.

81. Davidson RJ. Affective style and affective disorders: perspectives from affective neuroscience. Cogn Emot. 1998;12(3):307-30.

82. Davidson RJ. EEG measures of cerebral asymmetry: conceptual and methodological issues. Int J Neurosci. 1988;39(1-2):71-89.

83. Harmon-Jones E, Allen JJB. Behavioral activation sensitivity and resting frontal EEG asymmetry: covariation of putative indicators related to risk for mood disorders. J Abnorm Psychol. 1997;106(1):159-63.

84. Harmon-Jones E. Contributions from research on anger and cognitive dissonance to understanding the motivational functions of asymmetrical frontal brain activity. Biol Psychol. 2004;67(1-2):51-76.

85. Harmon-Jones E, Gable PA, Peterson CK. The role of asymmetric frontal cortical activity in emotion-related phenomena: A review and update. Biol Psychol. 2010;84(3):451-62.

86. Wilkowski BM, Meier BP. Bring it on: angry facial expressions potentiate approach-motivated motor behavior. J Pers Soc Psychol. 2010;98(2):201-10.

87. Harmon-Jones E, Allen JJB. Anger and frontal brain activity: EEG asymmetry consistent with approach motivation despite negative affective valence. J Pers Soc Psychol. 1998;74(5):1310-6.

88. Flagg EJ, Cardy JEO, Roberts W, Roberts TPL. Language lateralization development in children with autism: insights from the late field magnetoencephalogram. Neurosci Lett. 2005;386(2):82-7.

89. Herbert MR. Brain asymmetries in autism and developmental language disorder: a nested whole-brain analysis. Brain. 2004;128(1):213-26.

90. Popescu M, Popescu EA, Chan T, Blunt SD, Lewine JD. Spatio-temporal reconstruction of bilateral auditory steady-state responses using MEG beamformers. IEEE Trans Biomed Eng. 2008;55(3):1092-102.

91. Farran EK, Branson A, King BJ. Visual search for basic emotional expressions in autism; impaired processing of anger, fear and sadness, but a typical happy face advantage. Res Autism Spectr Disord. 2011;5(1):455-62.

92. Kuusikko S, Haapsamo H, Jansson-Verkasalo E, Hurtig T, Mattila M-L, Ebeling $\mathrm{H}$, et al. Emotion recognition in children and adolescents with autism spectrum disorders. J Autism Dev Disord. 2009:39(6):938-45.

93. Markham R, Adams K. The effect of type of task on children's identification of facial expressions. J Nonverbal Behav. 1992;16(1):21-39.

94. De Sonneville LMJ, Verschoor C a, Njiokiktjien C, Op het Veld V, Toorenaar N, Vranken M. Facial identity and facial emotions: speed, accuracy, and processing strategies in children and adults. J Clin Exp Neuropsychol. 2002;24(2):200-13.

95. Vicari S, Reilly JS, Pasqualetti P, Vizzotto A, Caltagirone C. Recognition of facial expressions of emotions in school-age children: the intersection of perceptual and semantic categories. Acta Paediatr. 2000;89(7):836-45.

96. Herba C, Phillips M. Annotation: development of facial expression recognition from childhood to adolescence: behavioural and neurological perspectives. J Child Psychol Psychiatry. 2004;45(7):1185-98.

97. Olofsson JK, Nordin S, Sequeira H, Polich J. Affective picture processing: an integrative review of ERP findings. Biol Psychol. 2008;77(3):247-65.

98. Güntekin B, Basar E. Emotional face expressions are differentiated with brain oscillations. Int J Psychophysiol. 2007;64(1):91-100. 
99. Scheeringa R, Fries P, Petersson K-M, Oostenveld R, Grothe I, Norris DG, et al. Neuronal dynamics underlying high- and low-frequency EEG oscillations contribute independently to the human BOLD signal. Neuron. 2011;69(3):572-83.

100. Waldhauser GT, Johansson M, HansImayr S. Alpha/beta oscillations indicate inhibition of interfering visual memories. J Neurosci. 2012;32(6):1953-61.

101. Engel AK, Fries P. Beta-band oscillations-signalling the status quo? Curr Opin Neurobiol. 2010;20(2):156-65.

102. Perry A, Troje NF, Bentin S. Exploring motor system contributions to the perception of social information: Evidence from EEG activity in the mu/ alpha frequency range. Soc Neurosci. 2010;5(3):272-84.

103. Wright B, Alderson-Day B, Prendergast G, Bennett S, Jordan J, Whitton C, et al. Gamma activation in young people with autism spectrum disorders and typically-developing controls when viewing emotions on faces. Koenig T, editor. PLoS One. 2012;7(7):e41326.

104. Hinojosa JA, Mercado F, Carretié L. N170 sensitivity to facial expression: a meta-analysis. Neurosci Biobehav Rev. 2015;55:498-509.

105. Zion-Golumbic E, Golan T, Anaki D, Bentin S. Human face preference in gamma-frequency EEG activity. Neuroimage. 2008;39(4):1980-7.

106. Gao Z, Goldstein A, Harpaz Y, Hansel M, Zion-Golumbic E, Bentin S. A magnetoencephalographic study of face processing: M170, gamma-band oscillations and source localization. Hum Brain Mapp. 2013;34(8):1783-95.

\section{Submit your next manuscript to BioMed Central} and we will help you at every step:

- We accept pre-submission inquiries

- Our selector tool helps you to find the most relevant journal

- We provide round the clock customer support

- Convenient online submission

- Thorough peer review

- Inclusion in PubMed and all major indexing services

- Maximum visibility for your research

Submit your manuscript at www.biomedcentral.com/submit 